\title{
Evaluation of Anal Cytology in Women with History of Abnormal Pap Smear, Cervical Intraepithelial Neoplasia, Cervical Cancer and High Risk HPV for Anogenital Dysplasia
}

\author{
Maryam Sadat Hosseini ${ }^{1}$, Donya Khosravi ${ }^{1 *}$, Farah Farzaneh ${ }^{1}$, Abdalali \\ Ebrahimi ${ }^{2}$, Maliheh Arab ${ }^{1}$, Tahereh Ashraf Ganjoie ${ }^{1}$, Farzane Jamdar ${ }^{1}$, Atefeh \\ Moridi ${ }^{1}$, Mohammad Chehrazi ${ }^{3}$
}

\begin{abstract}
Background: Anal cancer is uncommon. Although uncommon, the incidence of anal cancer has increased among the general population of the United States and other countries over the past 30 years. We evaluated anal cytology in women with the history of abnormal pap smear, cervical intraepithelial neoplasia, cervical cancer and high risk HPV for anogenital dysplasia. Methods: In this cross-sectional study, 153 patients over the age of 21 years, referred to Imam Hossein Hospital in 1395-1396 who were being monitored due to abnormal pap smear, cervical cancer, CIN and high risk HPV, were evaluated with anal cytology for anogenital dysplasia. Results: 153 patients were enrolled in four main groups. Among those with a history of abnormal pap smear (39 patients), only $25 \%$ of the patients with HSIL had abnormal anal pap smear, and the rest of them had negative anal smear. Among those with a history of CIN (61 patients), patients with CIN III, 30.8\% had abnormal anal smear, while the rest had a negative anal smear. Among those with a high risk of HPV (23 patients), 50\% of the patients with concurrent positive HPV 16 and 18 had anal abnormal smear. Patients with a history of cervical cancer (30 patients) who had radiotherapy with or without surgery showed a negative anal smear. The ratio of anal SIL positive of individuals with a sexual partner was only $1.9 \%$, while this ratio was $27.3 \%$ for those with more than one partner and this difference was statistically significant $(\mathrm{P}<0.0001) .15 .4 \%$ of smokers had positive anal SIL test result, while, only $2.9 \%$ non-smokers had the same result, and this difference was statistically significant $(\mathrm{P}=0.03) .20 \%$ of the patients who had anal intercourse showed a positive anal SIL score result, compared to $1 \%$ for those who did not. This difference was statistically significant $(\mathrm{P}<0.0001)$. Conclusion: Among people with a history of abnormal cervical pap smear, CIN, cervical cancer and high-risk HPV during the last 6 months, abnormal anal tests are most commonly seen in patients who had risk factors such as anal sex, cigarette, multi partner etc. with high grade interepithelial squamous cervix such as HSIL, CIN III, HPV 16 and 18; more studies in larger sample size are needed to have the better conclusion.
\end{abstract}

Keywords: Anal neoplasms- cervical cancer- anal canal abnormalities- papanicolaou smear

Asian Pac J Cancer Prev, 19 (11), 3071-3075

\section{Introduction}

Cervical and anal cancers have the same pathological, histological and embryological characteristics. Both of them originate from embryonic cloaca and are formed of a squamous and cylindrical epithelial joint. Both areas may show HPV-related normal metaplastic and abnormal dysplasia changes.

Anal cancer is uncommon. Although uncommon, the incidence of anal cancer has increased among the general population of the United States and other countries over the past 30 years (Goldman et al., 1989; Frisch et al.,
1993; Johnson et al., 2004; Chiao et al., 2005; Brewster and Bhatti, 2006). Increased incidence is associated with: female gender, HPV infection, multipartner, genital warts, cigarettes, anal intercourse and HIV-positive (Palefsky, 1994). In specific populations such as homosexual men, there is an increased incidence of anal cancer (Daling et al., 1982). The role of sexual activity in the development of anal cancer is due to reports that have proven strong links between the anal and cervical cancers (Peters et al., 1984; Melbye and Sprøgel, 1991; Frisch et al., 1994). HPV is one of the most common STD infections which is strongly associated with sexual activity and anal cancer

${ }^{1}$ Preventative Gynecology Research Center, ${ }^{2}$ Department of Pathology, Imam Hossein Hospital, Shahid Beheshti University of Medical Sciences, ${ }^{3}$ Department of Epidemiology and Reproductive Health, Reproductive Epidemiology Research Center, Royan Institute for Reproductive Biomedicine, ACECR, Tehran, Iran. *For Correspondence:dr.khosravi.d@gmail.com 
(Northfelt et al., 1996; Frisch et al., 1997).

In the United States, the incidence of SCC from 1973 to 2009 showed 3 and 1.7 times increase in males and females, respectively (Machalek et al., 2012). In the past, it has been thought that anal cancer is more likely to occur in cases where a person is under inflammatory conditions such as fissure, fistula and hemorrhoids. However, subsequent case-control studies have shown that they had no effects on the development of anal cancers (Kline et al., 1964; Holly et al., 1989).

In the current study, we aimed to examine the anal of people who had already been examined for cervical disorder and diagnosed to be at high risk, which is because of the common cause of cervix and anal in creating premalignant and malignant cases, as well as important risk factors such as anal-Intercourse and multipartner, and cultural problems associated with the precise recording of the history of sexual relationships in all societies, including Iran, in the case of people who have been examined for cervical disorder and have been at high risk.

\section{Materials and Methods}

153 patients over the age of 21 years, referred to Imam Hossein Hospital in 1395-1396 who were being monitored due to abnormal pap smear, cervical cancer, $\mathrm{CIN}$ and high risk HPV, were entered into a cross-sectional study. Exclusion criteria were: colorectal cancer history, cognitive impairment, and pregnancy. After approval by the ethics committee of the University of Shahid Beheshti, formal consents were obtained from all patients. The anal cytology was performed using the liquid based method by inserting a brush into the anal hole and rotating it around 3 to 5 times in order to obtain sufficient cells for cytology study and were sent to a laboratory. Thereafter, a standard questionnaire was prepared by the first author, which included the following items: anal intercourse, number of sexual partner, genital wart, history of cigarette smoking, first cuitus and itch. After preparation of the test results, people with abnormal anal cytology were referred to a colorectal oncology fellowship for anal anoscopy and biopsy.

Quantitative data are presented using mean and standard deviations, and qualitative data are presented using numbers and percentages. Estimated prevalence was displayed with $95 \%$ confidence interval. ANOVA and Chi-square tests were used to compare quantitative and qualitative variables between groups with abnormal cervical. The significance level was set at $\mathrm{P}<0.05$ for all tests and SPSS software v. 21 was used for data analysis.

\section{Results}

Table 1 shows the status of abnormal pap smear, CIN, cervical cancer and high risk HPV in both positive and negative anal SAL and also in general. $25 \%$ of the patients who had HSIL pap smear also had positive anal SIL test, which had a significant difference with normal pap smear group $(\mathrm{P}=0.01)$. Those with ASCUS, LSIL, or ASC-H Pap smear test results had no positive SILI results. Regarding the CIN results, $30.8 \%$ of the patients with CIN III had positive anal SIL results, and this ratio had a significant difference with the normal group $(\mathrm{P}<0.0001)$. Furthermore, none of them in the CIN I or CIN II groups had positive anal tests. None of the patients with cervical cancer had positive anal test, but $5.4 \%$ of those without this type of cancer had a positive anal SIL result, which was not statistically significant $(\mathrm{P}=0.22) .50 \%$ of the people who had the same positive HPV 16 and 18 showed a positive result for anal SIL test and had a statistically significant difference with the group with no high risk of HPV $(\mathrm{P}=0.001)$. Moreover, those who had HPV 18 or

Table 1. Results of anal Cytology in Four Main Ggroup

\begin{tabular}{|c|c|c|c|c|c|c|}
\hline Variable & Total & Positive Anal SIL (\%) & Negative Anal SIL (\%) & PR & $95 \% \mathrm{CI}$ & $\mathrm{P}$ value \\
\hline \multicolumn{7}{|l|}{ Abnormal } \\
\hline Ascus & $16(11.4)$ & $0(0 \%)$ & $13(100 \%)$ & 1.04 & $0.99-1.07$ & 0.49 \\
\hline LSIL & $8(5.7 \%)$ & $0(0 \%)$ & $8(100 \%)$ & 1.04 & $0.99-1.07$ & 0.59 \\
\hline HSIL & $8(5.7 \%)$ & $2(25 \%)$ & $6(75 \%)$ & 6.94 & $1.36-35.71$ & 0.01 \\
\hline \multicolumn{7}{|l|}{ Pap smear } \\
\hline ASC-H & $7(5 \%)$ & $0(0 \%)$ & $5(100 \%)$ & 1.04 & $0.99-1.07$ & 0.66 \\
\hline \multicolumn{7}{|l|}{$\mathrm{CIN}$} \\
\hline CIN I & $31(22.1 \%)$ & $0(0 \%)$ & $22(100 \%)$ & 1.01 & $0.99-1.04$ & 0.57 \\
\hline CIN II & $17(12.1 \%)$ & $0(0 \%)$ & $13(100 \%)$ & 1.01 & $0.99-1.04$ & 0.66 \\
\hline CIN III & $13(9.3 \%)$ & $4(30.8 \%)$ & $9(69.2 \%)$ & 21.74 & $2.61-166.66$ & $<0.0001$ \\
\hline \multicolumn{7}{|l|}{ Cervical Cancer } \\
\hline SCC & $30(21.4 \%)$ & $0(0 \%)$ & $26(100 \%)$ & 1.06 & $1.01-1.11$ & 0.22 \\
\hline HPV 16 & $15(10.7 \%)$ & $1(7.7 \%)$ & $12(92.3 \%)$ & 2.51 & $0.28-22.22$ & 0.4 \\
\hline HPV 18 & $1(7 \%)$ & $0(0 \%)$ & $1(100 \%)$ & 1.03 & $1.00-1.07$ & 0.86 \\
\hline HPV 16,18 & $3(2.1 \%)$ & $1(50 \%)$ & $1(50 \%)$ & 16.39 & $2.76-100$ & 0.001 \\
\hline others & $4(2.9 \%)$ & $0(0 \%)$ & $4(100 \%)$ & 1.03 & $1.00-1.07$ & 0.72 \\
\hline
\end{tabular}

SD, Standard Deviation; PR, Prevalence Ratio; CI, Confidence Interval; Atypical squamous cells of undetermined significance (ASC-US); Lowgrade squamous intraepithelial lesion (LSIL); High-grade squamous intraepithelial lesions (HSIL); Atypical squamous cells: cannot exclude highgrade squamous intraepithelial lesion (ASC-H). 
Table 2. Demographic Variables and Risk Factors of Abnormal Pap Smear, CIN and HPV

\begin{tabular}{|c|c|c|c|c|c|c|}
\hline Variable & Total & Negative Anal SIL (\%) & Positive Anal SIL (\%) & PR & CI $95 \%$ & $\mathrm{P}$-value \\
\hline Age & 42.63 & $42.47(\mathrm{sd}=11.37)$ & $33.6(\mathrm{sd}=10.36)$ & $\mathrm{ES}=0.03$ & $40.76-44.50$ & 0.09 \\
\hline \multicolumn{7}{|l|}{ Partner } \\
\hline 1 & $128(91.4 \%)$ & $105(98.1 \%)$ & $2(1.9 \%)$ & & & \\
\hline More than 1 & $12(8.6 \%)$ & $8(72.7 \%)$ & $3(27.3 \%)$ & 14.49 & $2.072-76.92$ & $<0.0001$ \\
\hline \multicolumn{7}{|l|}{ Partner of spouse } \\
\hline 1 & $120(85.7 \%)$ & $98(98 \%)$ & $2(2 \%)$ & & & \\
\hline More than 1 & $20(14.3 \%)$ & $15(83.3 \%)$ & $3(16.7 \%)$ & 8.33 & $1.50-45.45$ & 0.004 \\
\hline Non-smoker & $126(90 \%)$ & $102(97.1 \%)$ & $3(2.9 \%)$ & & & \\
\hline Smoker & $14(10 \%)$ & $11(84.6)$ & $2(15.4 \%)$ & 5.38 & $1.01-29.41$ & 0.03 \\
\hline \multicolumn{7}{|l|}{ Anal intercourse } \\
\hline No & $117(83.6 \%)$ & $97(99 \%)$ & $1(1 \%)$ & & & \\
\hline Yes & $23(16.4 \%)$ & $16(80 \%)$ & $4(20 \%)$ & 19.61 & $2.31-166.66$ & $<0.0001$ \\
\hline Age of First Cuitus & 19.69 & $19.58(\mathrm{sd}=3.72)$ & $20.60(\mathrm{sd}=3.05)$ & $\mathrm{ES}=0.004$ & $-2.32,4.37$ & 0.54 \\
\hline \multicolumn{7}{|l|}{ Genital Wart } \\
\hline No & $107(76.4 \%)$ & $86(97.7 \%)$ & $2(2.3 \%)$ & & & \\
\hline Yes & $33(23.6 \%)$ & $27(90 \%)$ & $3(10 \%)$ & 4.41 & $0.77-25$ & 0.07 \\
\hline \multicolumn{7}{|l|}{ Itch } \\
\hline No & $95(67.9 \%)$ & $79(96.3 \%)$ & $3(3.7 \%)$ & & & \\
\hline Yes & $45(32.1 \%)$ & $34(94.4 \%)$ & $2(5.6 \%)$ & 1.52 & $0.26-8.69$ & 0.64 \\
\hline
\end{tabular}

SD, Standard Deviation; ES, Effect Size; PR, Prevalence Ratio

other subjects did not have any positive results for their anal SIL test. $7.7 \%$ of the patients in the HPV16 group showed a positive anal SIL test result but did not show a significant difference with the normal group $(\mathrm{P}=0.4)$.

Table 2 shows the demographic variables and patient risk factors. The mean age of the individuals was 42.63 years, which was $33.6(\mathrm{sd}=10.36)$ and $42.47(\mathrm{sd}=11.37)$ in the positive and negative anal SIL groups, respectively $(\mathrm{P}=0.09)$, and did not have a significant difference $(\mathrm{P}=0.09)$. The ratio of anal SIL positive of individuals with a sexual partner was only $1.9 \%$, while this ratio was $27.3 \%$ for those with more than one partner and this difference was statistically significant $(\mathrm{P}<0.0001)$. Also, these ratios for their husbands were $2 \%$ and $16.7 \%$, respectively, which were statistically significant $(\mathrm{P}=0.004) .15 .4 \%$ of smokers had positive anal SIL test result, while, only $2.9 \%$ non-smokers had the same result, and this difference was statistically significant $(\mathrm{P}=0.03)$. $20 \%$ of the patients who had anal intercourse showed a positive anal SIL score result, compared to $1 \%$ for those who did not. This difference was statistically significant $(\mathrm{P}<0.0001)$.

The average age of the first intercourse was 19.69 years. The average was 20.6 and 19.58 years in positive and negative anal SIL groups, respectively, which did not show any statistical significance $(\mathrm{P}=0.54)$. The ratio of positive anal SIL in people with genital warts was $10 \%$ and $2.3 \%$ for those who did not have a positive anal SIL. Although there was a significant difference $(P=0.07)$ between these groups. Moreover, the ratio of positive anal SIL among those with or without itching was 5.6\% and $3.7 \%$, respectively, and this difference was not statistically significant $(\mathrm{P}=0.64)$.

\section{Discussion}

In this study, 153 patients were enrolled in four main groups. Among those with a history of abnormal pap smear (39 patients), only $25 \%$ of the patients with HSIL had abnormal anal pap smear, and the rest of them had negative anal smear. Among those with a history of CIN (61 patients), patients with CIN III, 30.8\% had abnormal anal smear, while the rest had a negative anal smear. Among those with a high risk of HPV (23 patients), 50\% of the patients with concurrent positive HPV 16 and $18 \mathrm{had}$ anal abnormal smear. Patients with a history of cervical cancer (30 patients) who had radiotherapy with or without surgery showed a negative anal smear.

In a study by Colare et al., (2010) on 49 patients with abnormal anal smear showed that the prevalence of cytological abnormal anal will be higher in cases with a high grade cervical pap smear (such as HSIL). However, anal smear sample was prepared less than a week from cervical cytology sampling. They also concluded that anal mucus is a HPV reservoir that can be a source of cervical reinfection.

In this study, the anal test was carried out on individuals with a history of abnormal cervical over the past 6 months and had undergone the test for their usual treatments, which could be the cause of difference in the incidence of further anal abnormal tests in other studies with this study, and the results of this study also indicated that in people with high grade squamous cervix, the likelihood of lesion occurring in anal is more than low grade cases.

In this study, it was shown that risk factors such as multipartner, cigarette, anal sex and genital warts were involved in individuals who had anal abnormal tests and 
were different from those who had a negative anal test, which was statistically significant $(\mathrm{P}<0.05)$. In contrast, in a study by Nobre et al., (2016), which was carried out on two groups of healthy screening and people with ascus pap smear cervix and over, they showed that none of the risk factors such as cigarettes, partner numbers, anal sex, etc. had a statistically significant difference between these groups. However, the difference between two studies can be due to the sampling methods, which in our study healthy individuals were not included in the study after screening.

In a study by Jacyntho et al., (2011), 184 patients with genital SIL and 76 controls without a prior genital SIL showed that $32(17.4 \%)$ out 184 patients with a genital history of SIL compared with $2(6.2 \%)$ out of 76 controls had an abnormal anal test.

Several studies (Kost et al., 2012; Tatti et al., 2012) have reported that HIV-infected women have higher levels of abnormal anal tests than HIV negative ones. In this study, no patient was HIV-infected.

In another study (Saleem and Paulus), the long-term risk of secondary cancers among 104,760 patients with cervical cancer was examined and the results showed that women with a history of cervical cancer are at greater risk of developing anal cancer than general population.

In our study, abnormal anal test was detected in none of the cervical cancer patients, which may be due to the low number of cervical cancer samples in this study compared to other studies.

As shown in other studies (Kost et al., 2012; Tatti et al., 2012; Arora et al., 2014), the anal pap smear test is a useful tool for early diagnosis of anal dysplasia in people with high grade and risk cervical lesions. In our study, this finding was confirmed and we showed that the anal abnormal test in patients with high grade cervical lesions had a higher rate.

In a study by Shvetsov et al., (2009) there are reportson the recurrence of HPV anal infection or abnormal anal test in a few months with the average clearance of 132 and 212 days for HPV 16 and HPV 18, respectively.

In conclusion, among people with a history of abnormal cervical pap smear, CIN, cervical cancer and high-risk HPV during the last 6 months, abnormal anal tests are most commonly seen in patients who had risk factors such as anal sex, cigarette, multi partner etc. with high grade interepithelial squamous cervix such as HSIL, CIN III, HPV 16 and 18. In other low-grade patients with cervical lesions, the anal tests were mostly negative.

\section{Acknowledgements}

We would like to thank the nursing, the administrative and secretarial staff of the obstetrics and gynecology department and clinic at Imam Hossein hospital for their contribution to the maintenance of our patient record without which this project would have been impossible.

\section{References}

Arora R, Pandhi D, Mishra K, et al (2014). Anal cytology and p16 immunostaining for screening anal intraepithelial neoplasia in HIV-positive and HIV-negative men who have sex with men: a cross-sectional study. Int J STD AIDS, 25, 726-33.

Brewster DH, Bhatti LA (2006). Increasing incidence of squamous cell carcinoma of the anus in Scotland, 1975-2002. Br J Cancer, 95, 87-90.

Chaves EBM, Folgierini H, Capp E, et al (2012). Prevalence of abnormal anal cytology in women infected with HIV. J Med Virol, 84, 1335-9.

Chiao EY, Krown SE, Stier EA, et al (2005). A population-based analysis of temporal trends in the incidence of squamous anal canal cancer in relation to the HIV epidemic. J Acquir Immune Defic Syndr, 40, 451-5.

Colare EE, Giaccio CMS, Nadal SR (2010). Prevalence of Anal cytology abnormalitiesin women with positive cervical cytology. Diagn Cytopathol, 39, 323-7.

Daling JR, Weiss NS, Klopfenstein LL, et al (1982). Correlates of homosexual behavior and the incidence of anal cancer. JAMA, 247, 1988-90.

Frisch M, Glimelius B, van den Brule AJ, et al (1997). Sexually transmitted infection as a cause of anal cancer. $N$ Engl $J$ Med, 337, 1350-8.

Frisch M, Melbye M, Møller H (1993). Trends in incidence of anal cancer in Denmark. BMJ, 306, 419-22.

Frisch M, Olsen JH, Melbye M (1994). Malignancies that occur before and after anal cancer: clues to their etiology. Am J Epidemiol, 140, 12-9.

Goldman S, Glimelius B, Nilsson B, et al (1989). Incidence of anal epidermoid carcinoma in Sweden 1970-1984. Acta Chir Scand, 155, 191-7.

Holly EA, Whittemore AS, Aston DA, et al (1989). Anal cancer incidence: genital warts, anal fissure or fistula, hemorrhoids, and smoking. J Natl Cancer Inst, 81, 1726-31.

Jacyntho PC, Horta AA, A CM (2011). Association between genital intraepithelial lesion and anal squamous intraepithelial lesion in HIV negative women. Am J Obstet Gynecol, 1155, 205.

Johnson LG, Madeleine MM, Newcomer LM, et al (2004). Anal cancer incidence and survival: the surveillance, epidemiology, and end results experience, 1973-2000. Cancer, 101, 281-8.

Kline RJ, Spencer RJ, Jr HE (1964). Carcinomaassociated with fistula-in-ano. Arch Surg, 89, 989-94.

Kost B Gingel, Air A, Weissenbacher T (2012). Anal cytology as a screening tool for early detection of anal dysplasia in HIV-infected women. Anticancer Res Zoll, 30, 1719-23.

Machalek DA, Poynten M, Jin F, et al (2012). Anal human papillomavirus infection and associated neoplastic lesions in men who have sex with men: a systematic review and meta-analysis. Lancet Oncol, 13, 487-500.

Melbye M, Sprøgel P (1991). Aetiological parallel between anal cancer and cervical cancer. Lancet, 338, 657-9.

Nobre MDS, Jacyntho CM, Jr JE, et al (2016). Abnormal anal cytology risk in women with known genital squamous intraepithelial lesion. Braz J Infect Dis, 20, 294-7.

Northfelt DW, Swift PS, Palefsky JM (1996). Anal neoplasia. Pathogenesis, diagnosis, and management. Hematol Oncol Clin North Am, 10, 1177-87.

Palefsky JM (1994). Anal human papillomavirus infection and anal cancer in HIV-positive individuals: an emerging problem. AIDS, 8, 283-95.

Peters RK, Mack TM, Bernstein L (1984). Parallels in the epidemiology of selected anogenital carcinomas. $J$ Natl Cancer Inst, 72, 609-15.

Saleem AM, Paulus JK ShapterAP (2011). Risk of anal cancer in a cohort with human papillomavirus-ralated gynecologic neoplasm. Obstet Gynecol, 121, 643-9. 
Shvetsov YB, Hernandez BY, McDuffie K, et al (2009). Duration and clearance of anal human papillomavirus (HPV) infection among women: the Hawaii HPV cohort study. Clin Infect Dis, 48, 536-46.

Tatti S, Suzuki V, Fleider L, et al (2012). Anal intraepithelial lesions in women with human papillomavirus-related disease. J Low Genit Tract Dis, 16, 454-9.

\section{(1) $(8$}

This work is licensed under a Creative Commons AttributionNon Commercial 4.0 International License. 